\title{
Understanding Complexity of Mastocytosis
}

\author{
*Nahla AM Hamed \\ Professor of Hematology, Alexandria University, Egypt
}

Submission: July 04, 2017; Published: July 05, 2017

*Corresponding author: Nahla AM Hamed, Professor of Hematology, Faculty of Medicine, Alexandria University , Egypt, Email: drhamedn@hotmail.com

\section{Abstract}

SM is regarded as a stem cell-derived hematologic neoplasm that manifests in indolent variants and advanced entities. Does a single mutation in the KIT receptor can potentially contribute to such disease heterogeneity? Identifying other non-KIT associated mutations may contribute to the disease heterogeneity in these patients. However, how these mutations precisely contribute to disease pathogenesis remains an active area of investigation.

Abbreviation: MCs: Mast Cells; BM: Bone Marrow; GI: Gastro Intestinal; CM: Cutaneous Mastocytosis; SM: Systemic Mastocytosis; ISM: Indolent SM; SSM: Smoldering SM; ASM: Aggressive SM; MCL: Mast Cell Leukemia; SM-AHN: SM with associated Hematologic Neoplasm; TKIs: Tyrosine Kinase Inhibitors; S/A/Rpos: Mutation(s) in the SRSF2/ASXL1/RUNX1 Gene Panel; SAHA: Suberoyl Anilide Hydroxamic acid

\section{Introduction}

Mastocytosis results from abnormal proliferation of MCs and resultant infiltration of various organs [1]. The most commonly affected tissues are the skin, BM, and GI tract, whereas the liver, spleen, and lymph nodes are less commonly involved [2]. The estimated prevalence of mastocytosisis 13 per 100.000 in all age categories [1]. Mastocytosis is no longer considered a subgroup in the MPNs classification but now is a separate disease category due to its unique clinical and pathologic features [3].

The 2016 classification of the WHO divides the disease into cutaneous mastocytosis (CM), systemic mastocytosis (SM), and Mast cell sarcoma. CM includes maculopapular CM, diffuse CM, and localized mastocytoma of skin. SM is further divided into indolent SM (ISM), smoldering SM (SSM), and advanced SM variants, including aggressive SM (ASM), mast cell leukemia (MCL), and SM with associated hematologic neoplasm (SM-AHN) [4]. The name of the 2008 category of "systemic mastocytosis with associated clonal hematological non-mast-cell lineage disease" is now replaced by the 2016 category of "SM-AHN" [3].

\section{Diagnosis of SM}

requires at least one major and one minor criterion, or three minor criteria. The only major criterion of SM is the multifocal accumulation and clustering of MC (at least $15 \mathrm{MC}$ /cluster) in the BM or another extracutaneous organ. Minor criteria include an abnormal MC morphology, expression of CD2 and/or CD25 in MC in extracutaneous organs, an activating mutation in codon 816 of KIT (usually KIT D816V) in extra-cutaneous cells, and a basal serum tryptase level exceeding $20 \mathrm{ng} / \mathrm{ml}$ (in case of an associated hematologic non-MC lineage disease, this criterion is not valid) [4]. If SM is diagnosed, the next step is to evaluate its aggressiveness according to the signs of organ infiltration, with or without organ enlargement (B-findings) and dysfunction (C-findings) [5]. Absent B- and C-findings reflect an ISM [6] and $\geq 2$ B-findings and no C findings reflect SSM [5]. The presence of at least one $\mathrm{C}$-finding directs the diagnosis towards an advanced SM (ASM, SM-AHN or MCL) [6].

\section{B-findings include}

i. Infiltration grade (MC) in BM of more than $30 \%$ and serum tryptase of more than $200 \mathrm{ng} / \mathrm{mL}$,

ii. Dysmyelopoiesis, and

iii. Organomegaly without impairment of organ function. C-findings include cytopenias, osteolysis, malabsorption, and organomegaly with functional impairment of the organ/ tissue (hypersplenism, portal hypertension, ascites) [7].

ASM is diagnosed when MCs in BM smears represent less than $20 \%$ of total nucleated cells. MCL is diagnosed when MCs in BM smears represent $>20 \%$ of total nucleated cells (with or without circulating neoplastic MCs in the bloodstream) [6]. A rare SM variant that has been described is ISM with recurrent anaphylaxis or vascular collapse in the absence of skin lesions [4]. 


\section{Molecular pathogenesis}

MCs and their committed progenitors express the KIT receptor (CD117), which is a type III receptor tyrosine kinase [8]. The interaction between KIT and its ligand, stem cell factor, plays a central role in regulating proliferation, growth, differentiation, adhesion, chemotaxis, and survival of MCs [1]. A KIT D816V mutation has been found in approximately $90 \%$ of patients with SM, irrespective of WHO SM subtype [1]. The mutation burden differs significantly among patients with different WHOsubgroups [9].

Alternative mutations at position 816, e.g. D816H or D816Y, were identified in a significant proportion of KIT D816V negative patients with MCL [10]. Other KIT mutations (D820G, V559I) have also been reported in ASM patients [8]. The presence of additional mutations in these patients, may explain the aggressive nature of ASM, progression to MCL and perhaps resistance to TKIs [8]. It is recommended that other exons of KIT gene should be sequenced in KIT D816V negative patients with advanced SM particularly MCL [10].

Additional genetic lesions involving epigenetic regulation, RNA splicing, and/or transcription have been described [2]. These additional mutations are usually acquired by neoplastic (stem) cells prior to KIT D816V [10]. ASXL1, TET2, JAK2 V617F, RUNX1, and SRSF2, are present in over $60 \%$ of advanced SM patients [10]. A slightly lower rate of mutations in genes such as CBL (3.8\%- 20.5\%), DNMT3A (12\%), KRAS, NRAS, EZH2 (5.1\%) and ETV6 has been reported [8]. Patients with advanced SM could carry as many as 3 and in some cases more than 5 mutations [8]. The severity, prognosis and overall survival of patients with advanced SM might be related to the pattern of mutations that are acquired during the course of the disease [8].

In SM-AHN, additional drivers, mutations, and signaling pathways have been also or even exclusively expressed in the AHN portion of the disease [11]. CALR mutations, PDGFRA and PDGFRB mutations, or BCR/ABL1, are usually not found in SM-AHN [11]. It is hypothesized that KIT D816V is likely to be an early genetic hit in a 'stem cell' that gives rise to mast cells and cells of myeloid lineage, and is likely followed by a second hit in the AHN component [8]. Although MCs are derived from hematopoietic stem cells, little is known about LSCs in advanced SM and MCL [11].

\section{Clinical Manifestations}

Mastocytosis is mostly acquired, although rare familial cases have been reported. It affects both children and adults [8]. Cutaneous form is typical for pediatric patients [1] and mostly regresses during adolescence [8]. Most adult patients are diagnosed with ISM where skin is the predominant organ affected, while the aggressive forms of SM (ASM, MCL) are relatively rare [8]. Elderly patients exhibit less frequent skin involvement and/or limited disease. However, there is frequent association with other myeloid malignancies, probably due to the high frequency of aggressive and AHN forms of mastocytosis in the ageing population [2].

Clinical manifestations mostly depend on the overall MC burden, the organs and/tissues involved, and the effects of the MC mediators such as histamine, leukotrienes, tryptase and heparin [4]. The most prominent symptoms are flush, hypotension and anaphylactic reactions, associated with MC mediators release triggered by insect stings, drugs (including NSAIDs, anesthetics and antibiotics), foods and mechanical stimulation. Other common complaints include GI manifestations, osteopenia or osteoporosis, depression and neurological symptoms [1]. Mastocytosis is also associated with hematologic disorders such as myeloproliferative or myelodysplastic disorders and rarely lymphoproliferative [12].

Mastocytosis can be a cause of acquired clotting abnormalities, particularly during mast cell activation and degranulation. Clotting disorders range from simple ecchymosis tendency to severe/life-threatening bleeds. Blood group 0 and $\mathrm{vWF}$ disease increases the risk of mucocutaneous bleed during mast cell degranulation. No cases of thrombosis have been reported [5]. There is no contraindication to pregnancy when mastocystosis-related pathologies are under appropriate medical control. Undiagnosed and inappropriately treated mastocytosis may lead to severe pregnancy complications, including fetal demise [1].

\section{Prognosis}

A new risk score was proposed for patients with SM. This score includes clinical variables, e.g. splenomegaly and elevated alkaline phosphatase, as well as molecular markers, e.g. Mutations in the S/A/R gene panel (S/A/Rpos) [10]. $S / A / R$ gene panel have a strong adverse impact on disease phenotype and prognosis [10]. S/A/R is the most significant prognostic factor identified for MCL patients [10]. The presence of ASXL1 mutations, besides KIT and TET2 may also contribute to the prognosis and survival of mastocytosis patients [8]. In indolent forms, life expectancy is similar to that of the general population. In ASM and SM-AHN, life expectancy is significantly reduced with a respective median survival of 6.7 years and 4.4 years, depending on organ dysfunction as well as AHN type and progression. MCL usually presents with rapid organ failure and is associated with a catastrophic median survival of around 6 months [5].

\section{Treatment}

Management options are limited to symptomatic treatment [13]. Few case series have suggested a potential value of antiIgE therapy in the treatment of recurrent anaphylaxis [12]. Bisphosphonate therapy has to be considered for osteopenia, osteoporosis, or osteolysis [11]. Mast cell disease-controlling drugs, particularly steroids, were the only effective way to 
achieve hemostasis [5]. Aggressive mastocytosis requires cytoreductive treatment due to impairment of affected organs [1]. No generally accepted first-line standard therapy for advanced SM [11]. Interferon- $\alpha$ has shown encouraging results in more aggressive forms of the disease [8]. More or less specific targeted drugs including, cladribine, midostaurin, and the CD30-targeting antibody-conjugate brentuximab, have been established over the past few years [11]. There is no curative therapy, except for allogeneic stem cell transplantation, however the majority of ASM patients are elderly not eligible for stem cell transplant [13].

\section{TKIs}

Imatinib treatment has yielded improvement in MC burden and clinical symptoms in the following groups: those with a history of $\mathrm{CM}$ and harboring a germline mutation in the KIT receptor (F522C) [8], presence of the mutant form K509I [11], most mutations in ECD of the KIT receptor, especially in pediatric patients, MCL patient with an ECD KIT mutation (A502-Y503dup) and patients with WT KIT. Imatinib resistance was demonstrated in patients with KIT mutations in the second catalytic domain (D816V/H/Y/N) due to disruption of the conformation of the KIT D816V mutant receptor, rendering the receptor permanently active, and cannot be targeted by Imatinib [8]. KIT D816V confers resistance against masitinib [11]. Dasatinib has yielded disappointing results in clinical trials harboring KIT D816V mutations [8].

Cladribine (2CdA) may be indicated in some patients with (progressive) SSM, to decrease the risk of severe life-threatening anaphylaxis by reducing the MC burden and the risk of severe mediator-related events. Whether midostaurin exerts similar beneficial effects in SSM remains unknown [11]. Cladribine induces clinically meaningful responses in about $50 \%$ of all patients with advanced SM; however, many patients develop resistance [11].

Midostaurin (PKC412) is a potent multikinase inhibitor of PKC, VEGFR-2, PDGFR, FLT3 and KIT [8]. It shows an overall response rate of $60 \%$ in aggressive SM [13]. In several clinical trials, it reduces MC burden in ASM and MCL [8] but is unable to eradicate the disease in advanced SM and relapses are seen quite frequently [11]. It may be useful in the context of HSCT, for bridging patients who are in need of debulking or as maintenance therapy after HSCT. In advanced SM, midostaurin may be combined with cladribine or with targeted antibodies that exert reasonable effects on neoplastic cells [11].

Allogeneic hematopoietic stem cell transplantation is an important approach in young and fit advanced SM, including MCL who have a suitable transplant donor. Transplant-related mortality risk is rather high in elderly patients ( $>60$ years), and in patients suffering from certain co morbidities (eg, cardiovascular, metabolic) [11]. In SM-AHN, the SM component should be managed as if no AHN was detected, and the AHN should be treated as if no SM was found, with recognition that in the SM context, any AHN counts as secondary [11].

There are no distinct parameters to predict the response to treatment and the risk for progression to SMCL or MCL-SAML. KIT D816V allele burden is a valuable follow-up parameter in untreated and drug-treated patients with advanced SM [9]. Patients responding to cytoreductive therapy showed a significant decrease in the KIT D816V burden. Moreover, the mutation is of prognostic significance concerning survival [7]. Monitoring of serum tryptase is useful for follow-up of the disease [4]. The role of the "don't eat me" receptor IAP-1 (CD47), the multidrug resistance protein 1 (CD243), and the recently detected in neoplastic MCs, PD-L1 (CD274), in drug resistance in advanced SM is under investigation [11].

\section{Future Direction}

JAKs, HDAC and STAT5 may be new targets for more efficient therapies in the future

i. JAKs mutations (5.1\%-13\%) have been identified in mastocytosis. The role of JAK in phosphorylation of STAT proteins downstream of KIT D816V mutant bearing cells indicates that JAKs can be potential targets to treat mastocytosis [8].

ii. SAHA is a pan-inhibitor of class I and II HDACs, and also affects acetylation of non-histone proteins. KIT D816V mutation sensitized MC to HDACi mediated killing. SAHA may be of value as specific treatment for SM, although the specific mechanism of action requires further investigation [13].

iii. STAT5 function in neoplastic MCs is linked to the PI3K/ AKT signaling pathways. Designing STAT5 inhibitors to treat different forms of mastocytosisis a challenge [8].

\section{Conclusion}

An update in 2016 WHO classification, pathogenesis and therapeutic options of mastocytosis has been developed. Future trials therapy is in the way.

\section{References}

1. Ciach K, Niedoszytko M, Abacjew-Chmylko A, Pabin I, Adamski $\mathrm{P}$ et al. (2016) Pregnancy and delivery in patients with mastocytosis treated at the Polish Center of the European Competence Network on Mastocytosis (ECNM). PLoS ONE 11(1): e0146924.

2. Rouet A, Aouba A, Damaj G, Soucié E, Hanssens K, et al. (2016) Mastocytosis among elderly patients. A multicenter retrospective French study on 53 patients. Medicine 95(24): 24-30.

3. Arber DA, Orazi A, Hasserjian R, Thiele J, Borowitz MJ, et al. (2016) The 2016 revision to the World Health Organization classification of myeloid neoplasms and acute leukemia. Blood 127(20): 2391-2405.

4. Conde-Fernandes I, Sampaio R, Moreno F, Palla-Garcia J, dos Anjos Teixeira M, et al. (2017) Systemic mastocytosis with KIT V560G mutation presenting as recurrent episodes of vascular collapse: response to disodium cromoglycate and disease outcome. Allergy Asthma Clin Immunol 13: 21-30. 
5. Carvalhosa AB, Aouba A, Damaj G, Canioni D, Brouzes C, et al. (2015) A French national survey on clotting disorders in mastocytosis. Medicine 94(40): e1414.

6. Bibi S, Zhang Y, Hugonin C, Mangean MD, He L, et al. (2016) A new humanized in vivo model of KIT D816V+ advanced systemic mastocytosis monitored using a secreted luciferase. Oncotarget 7(50): 82985-83000.

7. González-de-Olano D, Matito A, Orfao A, Escribano L (2016) Advances in the understanding and clinical management of mastocytosis and clonal mast cell activation syndromes. F1000Research 5: 2666.

8. Chatterjee A, Ghosh J, Kapur R (2015) Mastocytosis- a mutated KIT receptor induced myeloproliferative disorder. Oncotarget 6(21): 18250-18264.

9. Hoermann G, Gleixner KV, Dinu GE, Kundi M, Greiner G, et al. (2014) The KIT D816V allele burden predicts survival in patients with mastocytosis and correlates with the WHO type of the disease. Allergy 69(6): 810-813.
10. Jawhar M, Schwaab J, Meggendorfer M, Naumann N, Horny H-P, et al. (2017) The clinical and molecular diversity of mast cell leukemia with or without associated hematologic neoplasm. Haematologica 102(6): 1035-1043.

11. Valent P, Akin C, Metcalfe DD (2017) Mastocytosis: 2016 updated WHO classification and novel emerging treatment concepts. Blood 129(11): 1420-1427.

12. Cardet JC, Akin C, Lee MJ (2013) Mastocytosis: update on pharmacotherapy and future directions. Expert OpinPharmacother 14(15): 2033-2045.

13. Lyberg K, Ali HA, Grootens J, Kjellander M, Tirfing M, et al. (2017) Histone deacetylase inhibitor SAHA mediates mast cell death and epigenetic silencing of constitutively active D816V KIT in systemic mastocytosis. Oncotarget 8(6): 9647-9659.

\section{Your next submission with Juniper Publishers will reach you the below assets}

- Quality Editorial service

- Swift Peer Review

- Reprints availability

- E-prints Service

- Manuscript Podcast for convenient understanding

- Global attainment for your research

- Manuscript accessibility in different formats

( Pdf, E-pub, Full Text, Audio)

- Unceasing customer service

Track the below URL for one-step submission https://juniperpublishers.com/online-submission.php 